\title{
The role of lipid droplets in metabolic disease in rodents and humans
}

\author{
Andrew S. Greenberg, ${ }^{1}$ Rosalind A. Coleman, ${ }^{2}$ Fredric B. Kraemer, ${ }^{3}$ James L. McManaman, ${ }^{4}$ \\ Martin S. Obin, ${ }^{1}$ Vishwajeet Puri, ${ }^{5}$ Qing-Wu Yan, ${ }^{1}$ Hideaki Miyoshi, ${ }^{6}$ and Douglas G. Mashek ${ }^{7}$
}

\begin{abstract}
1 Obesity and Metabolism Laboratory, Jean Mayer USDA Human Nutrition Research Center on Aging at Tufts University, Boston, Massachusetts, USA. 2Department of Nutrition, University of North Carolina at Chapel Hill, Chapel Hill, North Carolina, USA. ${ }^{3}$ Division of Endocrinology, Stanford University; VA Palo Alto Health Care System, Palo Alto, California, USA. ${ }^{4}$ Division of Reproductive Sciences, Department of Obstetrics and Gynecology, University of Colorado, Anschutz Medical Campus, Denver, Colorado, USA. 5Department of Medicine, Section of Endocrinology, Diabetes and Nutrition, Boston University School of Medicine, Boston, Massachusetts, USA. ${ }^{6}$ Department of Internal Medicine II, Hokkaido University Graduate School of Medicine,
\end{abstract} Sapporo, Japan. ${ }^{7}$ Department of Food Science and Nutrition, University of Minnesota, St. Paul, Minnesota, USA.

\begin{abstract}
Lipid droplets (LDs) are intracellular organelles that store neutral lipids within cells. Over the last two decades there has been a dramatic growth in our understanding of LD biology and, in parallel, our understanding of the role of LDs in health and disease. In its simplest form, the LD regulates the storage and hydrolysis of neutral lipids, including triacylglycerol and/or cholesterol esters. It is becoming increasingly evident that alterations in the regulation of LD physiology and metabolism influence the risk of developing metabolic diseases such as diabetes. In this review we provide an update on the role of LD-associated proteins and LDs in metabolic disease.
\end{abstract}

\section{Overview of lipid droplets}

Lipid droplets (LDs) are intracellular organelles that store neutral lipids within cells. Over the last two decades there has been a dramatic growth in our understanding of LD biology and, in parallel, our understanding of the role they play in health and disease. LDs regulate the storage and hydrolysis of neutral lipids, including triacylglycerol (TAG) and/or cholesterol esters. For example, adipocytes, the major reservoir of TAG in the body, store their TAG within LDs, and TAG storage in adipocytes is increased in obese animals and humans. The rates of adipocyte lipolysis in many obese individuals are constitutively increased, resulting in elevated levels of circulating fatty acids, which may be stored as TAG in LDs within skeletal muscle and liver. Both local and circulating free fatty acids are thought to be important etiologic agents in the development of insulin resistance, hyperlipidemia, inflammation, and hepatic steatosis (1-3). In this article, we will briefly review the basic characteristics of LDs and then focus on our present knowledge of the current view of the role of LDs in metabolic disease.

Cells have developed the capacity to store fatty acids as neutral lipids within LDs for several reasons. An important role of LDs in adipocytes is to store fatty acids as TAG to serve as a reservoir of energetic substrates that can be released when food is scarce. The detrimental effects associated with excess fatty acid entry into cells are often termed "lipotoxicity." Cells protect themselves from these effects by either oxidizing the fatty acids or sequestering them as TAG within LDs. Consistent with this hypothesis, activation of PPAR $\alpha$, which increases the expression of genes that encode oxidative proteins, also increases the expression of LDs and LD-associated proteins $(4,5)$. PPAR $\gamma$ and PPAR , along with other transcription factors, can also promote droplet formation $(4,6)$. As noted above, when fatty acids exceed the oxidative capacity of cells, they not only enhance LD formation, but may also induce apoptosis. An example of the protection LD formation provides was demonstrated in an experiment in which exogenous oleic acid added to fibroblasts deficient in diacylglycerol acyltransferase 1 (DGAT1)

Conflict of interest: The authors have declared that no conflict of interest exists. Citation for this article: J Clin Invest. 2011;121(6):2102-2110. doi:10.1172/JCI46069. promoted lipotoxic cell death. Expression of DGAT1, the terminal step in TAG synthesis, in fibroblasts channeled excess fatty acids into TAG and LD formation and protected against cell death (7).

The ability to store TAG in LDs is evolutionarily conserved and observed in yeast, plants, invertebrates, and vertebrates (8). The biogenesis and regulation of LDs is an area of intense research interest and is well reviewed elsewhere (9-11). The enzymes involved in TAG biogenesis are located primarily in the endoplasmic reticulum, mitochondria, and related organelles $(12,13)$. It has been suggested that the TAG of the nascent LD forms a lens within the hydrophobic environment of the ER bilayer (14-16). However, it should be noted that there are several conflicting theories about how the droplets form (17). Ultimately, the TAG droplet becomes coated with a monolayer of phospholipid and a cadre of proteins that stabilize it within the cytoplasm of cells (14). The growth of individual LDs within most cells results in a multilocular appearance, whereas in cells such as white adipocytes, a large unilocular droplet forms. It has become evident that the regulation of LD metabolism and physiology is extremely dynamic in nature. Two recent RNAi screens in Drosophila cells uncovered a diverse array of proteins and cellular organelles that participate in LD physiology $(18,19)$. In all, up to $1.5 \%$ of the fly genome has been implicated in LD physiology, and proteomic analyses of LDs from yeast, mouse mammary gland, Chinese hamster ovary cells, human hepatoma cells, human squamous epithelial carcinoma cells, mouse 3T3-L1 adipocytes, fly cells, and leukocytes have revealed the existence of many LD-associated proteins, including specific marker proteins, structural proteins, enzymes involved in various aspects of cholesterol and fatty acid metabolism, and proteins that function as regulators of membrane trafficking (20-32).

A critical catalyst in our understanding of LD biology came with the identification of the first LD-associated protein, perilipin 1 (PLIN1). PLIN1 was discovered during an experiment in which adipocytes were incubated with radiolabeled ${ }^{32} \mathrm{P}$ to label the cellular pool of ATP. When the adipocytes were treated with catecholamines to activate cAMP and cAMP-dependent PKA, autoradiogram analysis showed a protein that contained more than $95 \%$ of all the ${ }^{32} \mathrm{P}$ (33). This protein was named "perilipin" because both 
cell fractionation and immunohistochemical studies demonstrated that it localized, in the absence and presence of PKA activation, specifically at the surface of LDs. Tissue surveys revealed that PLIN1 is most highly expressed in adipocytes and to a lesser extent in steroidogenic tissues (34). Cloning of the cDNA for Plin 1 identified a major isoform, perilipin A (35), although other isoforms are expressed at lower levels $(35,36)$. In silico analysis revealed four additional LD-associated proteins with homology to the N-terminal region of Plin1: ADRP (also referred to as Plin2), TIP47 (Plin3), S3-12 (Plin4), and OXPAT (Plin5). These constitute the perilipin/ ADRP/TIP47 (PAT) family, which is conserved in a variety of organisms, from slime mold to mammals $(36,37)$. In this review we will focus on PLIN1, which is the most abundant and most thoroughly studied PAT protein in adipocytes (38-42), and on PLIN2 because of the number of studies investigating it in hepatic steatosis. One hallmark of LD proteins is that, in general, they associate with LDs in response to lipid loading of cells (43-45). However, LD proteins may have differential affinities for LDs. Plin 1 actually competes with Plin2 for localization on the droplet, thereby preventing Plin2 from associating with the LD and causing Plin2 to be degraded (38, $40)$. Finally, droplets may also be heterogeneous and contain various combinations of the PAT proteins (46).

Dynamic interactions between Plin 1 and other proteins that regulate lipolysis have been uncovered over the last several years. As we noted before, alterations in lipolysis can be detrimental to systemic metabolism. Hydrolysis of stored TAG to glycerol and fatty acids requires the sequential actions of adipose triglyceride lipase (ATGL) to hydrolyze TAG to diacylglycerol, hormone-sensitive lipase (HSL) to hydrolyze diacylglycerol, and monoacylglycerol lipase to hydrolyze monoacylglycerol to fatty acids and glycerol (47). ATGL is associated with the LD surface, perhaps because of its patatin protein domain, and bound to the G0S2 protein, which inhibits its hydrolytic activity (48). The protein CGI-58 is associated with the LD under basal conditions and can associate with ATGL and increase its catalytic activity by up to 20-fold (49). While a small amount of HSL may be associated with the LD in the absence of PKA activation, HSL is predominately located in the cytoplasm $(50,51)$. Monoacylglycerol lipase is thought to be constitutively activated in the absence of any phosphorylation. In the absence of PKA activation, Plin1 appears to reduce lipolysis by binding and sequestering CGI-58 and, possibly, a small amount of HSL on the droplet (51-53). Basal levels of lipolysis are predominately due to constitutive actions of ATGL $(54,55)$. PKA activation hyperphosphorylates Plin1, resulting in the release of CGI-58, which then binds to and increases ATGL hydrolytic activity on the droplet $(52,53)$. Thus, a reduction in Plin 1 would result in release of CGI-58, thereby allowing it to bind ATGL, increasing the catalytic activity of ATGL and accelerating the first step of lipolysis. Additionally, PKA phosphorylation of HSL causes the protein to translocate to the LD surface, where it binds hyperphosphorylated Plin1 and preferentially hydrolyzes diacylglycerol. There are likely other potential mechanisms and factors that regulate lipolysis. In this review we will discuss how several of these proteins are dysregulated in human disease.

Like the PAT proteins, the CIDE family of proteins also associates with and regulates LD physiology. The CIDE family consists of three proteins: Cidea, Cideb, and Cidec (also referred to as fatspecific protein 27 [FSP27]). The N-terminal region of the CIDE proteins shares homology with the two-subunit proteins of DNA fragmentation factor, which is involved in apoptosis (56). Addi- tionally, the Cide proteins have regions of homology with PLIN1, including a hydrophobic domain thought to be important for binding to the phospholipid monolayer of the droplet (57). Cidec was the first of this family to be identified as an LD-associated protein $(58,59)$. Subsequently, similar to PAT proteins, ectopic expression of Cide proteins promotes LD formation and reduces TAG hydrolysis $(57,60,61)$. Relevant to their homology to proteins associated with apoptosis, ectopic expression of CIDE members enhances rates of apoptosis. However, when CIDE-expressing cells are incubated with oleic acid to promote TAG storage and LD formation, the rates of apoptosis are significantly reduced, presumably because sequestration of the Cide proteins on the droplets blocks their proapoptotic function (60). Cidea in lean mice is expressed predominately in brown adipose tissue (BAT), but not in white adipose tissue (WAT) (62). Cidec in mice is predominately expressed in WAT, but has also been found in BAT (62-64) and lipid-laden hepatocytes $(6,56)$. In humans, the expression pattern is slightly different, with both CIDEC and CIDEA highly expressed in white adipocytes $(57,65)$.

\section{Role of LD proteins in obesity-associated insulin resistance, diabetes, and lipoatrophic diabetes}

$L D$ proteins in lipoatrophic diabetes. Plin 1 and members of the PAT family are critical regulators of lipid metabolism. In obesity, insulin resistance has been linked to increased rates of adipocyte lipolysis $(1,3,66,67)$ and increased expression of cytokines such as TNF- $\alpha$. One of the first clues in elucidating Plin 1 function was the observation that TNF- $\alpha$ reduces Plin 1 mRNA and protein expression while concomitantly increasing basal lipolysis (68). Constitutive overexpression of Plin 1 in cultured adipocytes blocks the ability of TNF- $\alpha$ to increase lipolysis, confirming a role for PLIN1 expression in regulating basal or constitutive lipolysis (69). Critical insights into the role of PLIN1 in systemic metabolism were demonstrated when two separate laboratories independently generated lines of Plin1-null mice; both groups observed that the mice were lean and developed systemic insulin resistance as they got older $(70,71)$. Studies comparing lipolysis in Plin1-null and wild-type mice revealed that Plin1-null adipocytes had increased rates of constitutive (unstimulated) lipolysis and reduced catecholamine-stimulated lipolysis (70). In these cells, Plin2 replaced Plin 1 protein on the LD, which suggests that Plin2 is less efficacious in blocking basal adipocyte lipolysis $(70,72)$. Nelifinavir, an anti-HIV drug, has been reported to promote lipodystrophy in humans (73), and we demonstrated that it specifically enhanced Plin1 degradation by a lysosomal pathway in mouse adipocytes, resulting in increased rates of constitutive basal lipolysis. These data demonstrate that Plin1 protein enhances catecholaminestimulated lipolysis and, importantly, that a reduction in Plin1 protein expression is associated with increased constitutive lipolysis, which can promote systemic insulin resistance. Recently, three individuals were reported to have one of two different heterozygous missense mutations in PLIN1, which altered the amino acids in the carboxy terminus of the protein. When ectopically expressed in cells, these mutant proteins were found to have reduced ability to block TAG hydrolysis, leading to increased rates of constitutive lipolysis as compared with wild-type PLIN1 (74). These subjects had partial lipodystrophy, with loss of adipose tissue in their femerogluteal region and lower limbs, along with Cushingoid or acromegaloid features, insulin-resistant diabetes, hypertriglyceridemia and hepatic steatosis. 


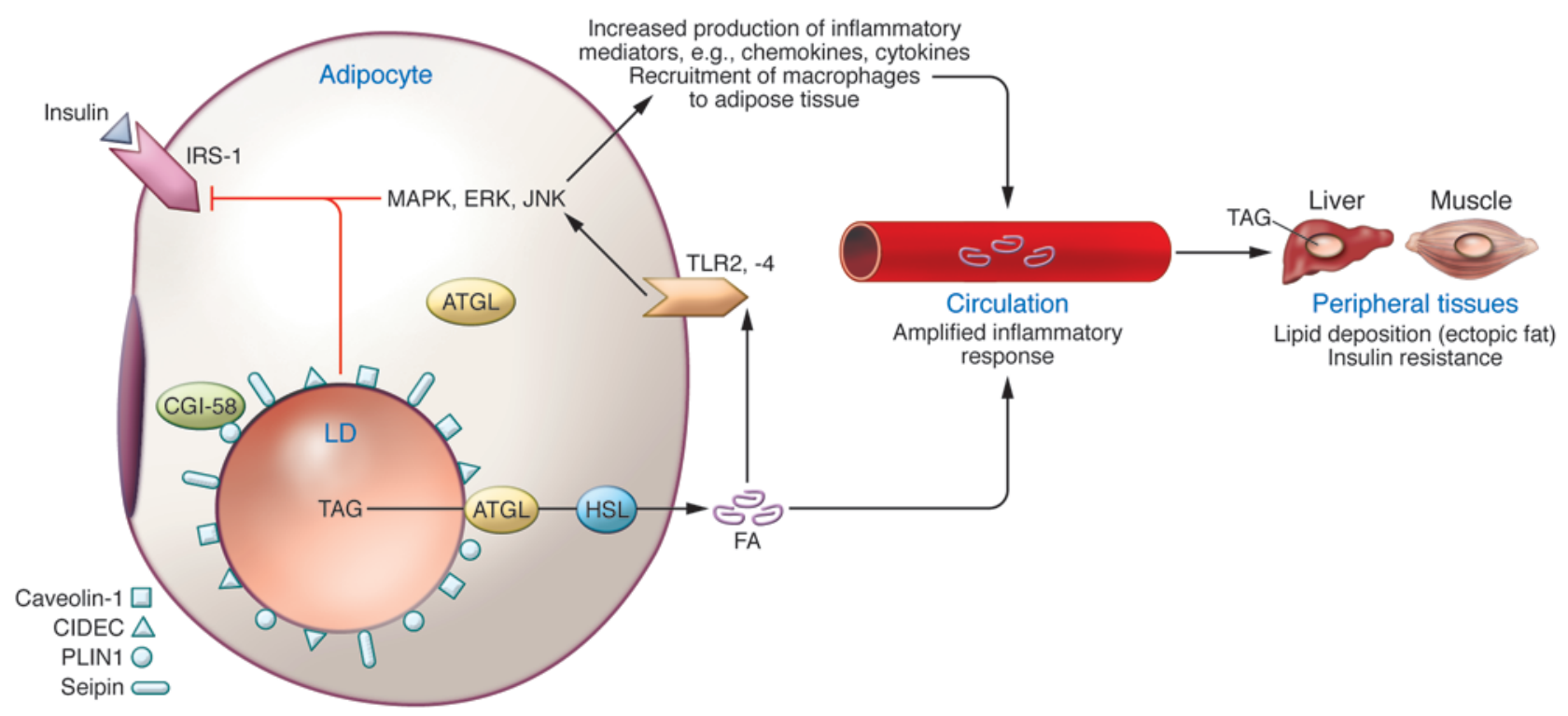

\section{Figure 1}

Alterations in LD proteins in adipocytes result in increased lipolysis and promote insulin resistance. In lipoatrophic and obese humans, reduced expression of PLIN1, CIDEC, or CIDEA in adipocytes results in constitutive release of fatty acids (FA). These fatty acids act locally and enter the bloodstream, where they activate inflammatory pathways, promote ectopic lipid deposition in peripheral tissues, and cause insulin resistance. The released fatty acids act locally to promote insulin resistance and inflammation by: (a) engaging TLRs, resulting in activation of MAPK signaling pathways and release of inflammatory mediators, which block insulin actions on lipolysis and glucose uptake; (b) recruiting macrophages that amplify the inflammatory response; and (c) inducing ectopic lipid deposition in hepatocytes and skeletal muscle cells and activating inflammatory pathways. A reduction in PLIN1 increases constitutive lipolysis by allowing CGI-58, normally associated with PLIN1, to activate LD-associated and non-LD-associated ATGL. Reductions in CIDEA or CIDEC enhance lipolysis by unclear mechanisms. In humans, genetic alterations in seipin and caveolin-1 reduce adipose tissue mass, resulting in lipodystrophy.

Similar to Plin1, TNF- $\alpha$ treatment of mouse adipocytes reduces Cidec expression, and siRNA knockdown of Cidec increases rates of basal constitutive lipolysis, whereas overexpression of Cidec blocks the effect of TNF- $\alpha$ on lipolysis $(57-60,65,75,76)$. Two separate laboratories generated Cidec-knockout mice $(63,64)$. Cidec-knockout mice have features similar to those of Plin1-null mice, such as reduced fat mass and increased lipolysis (63). The knockout animals have increased energy expenditure and are protected against the development of genetic or diet-induced obesity $(70,71)$. Basal lipolysis is increased in isolated white adipocytes from Cidec-null mice, and catecholamine- stimulated lipolysis is reduced (63). However, unlike Plin1-null mice, Cidec-null mice are protected against the development of diet-induced insulin resistance. One human patient has been described as having a homozygous nonsense mutation in the CIDEC gene that resulted in a truncated protein that was unable to associate with LDs (77). This patient had partial lipodystrophy with absence of femerogluteal and lower-limb adipose tissue, along with insulin-resistant diabetes, hypertriglyceridemia, and hepatic steatosis (see Figure 1). The mechanism by which Cidec blocks basal lipolysis remains poorly understood and is a goal of future research studies. Thus, loss of PLIN1 or CIDEC expression in humans, unlike mice, results in ectopic lipid accumulation, dyslipidemia, and diabetes.

Besides the increase in adipocyte lipolysis which reduces TAG content in adipocytes, the constitutive lipolysis induced by a reduction in either Plin 1 or Cidec also reduces fat mass by activating fat oxidation pathways. For example, fatty acids from lipolysis can directly activate transcription factors such as PPAR $\alpha$ to promote fat oxidation $(78,79)$. Additionally, lipolysis in white adipocytes can activate AMP-activated protein kinase, which also promotes fat oxidation and reduces lipogenesis (80). In both Cidec- and Plin1null mice, oxidative gene expression is increased in $\operatorname{WAT}(63,64,81$, 82). Furthermore, in Cidec-null mice, uncoupling protein 1 (UCP1) expression is increased in WAT, endowing it with characteristics of brown adipocytes such as multilocular LDs and enhanced thermogenesis (64). Thus, with reduced expression of LD proteins and increased lipolysis, fatty acids can promote fat oxidation. However, this compensatory pathway is not sufficient to prevent the detrimental release of fatty acids and subsequent development of insulin resistance, at least in Plin1-null mice. Potentially, differences in rates of constitutive lipolysis and fat oxidation in adipocytes may at least in part explain the differential susceptibility of Plin1- and FSP27/Cidec-null mice to insulin resistance. Of potential relevance is our observation that overexpressing human or mouse Plin 1 in female mice protects against the development of obesity and obesity-associated insulin resistance while increasing expression of oxidative genes in adipose tissue $(83,84)$. Overexpression of Plin1 potently reduces expression of FSP27 in adipocytes, which may partly explain the protection against obesity and insulin resistance. In summary, a significant reduction in LD proteins may promote both lipolysis and oxidative metabolism, providing another mechanism to reduce adipose mass.

While BAT has recently emerged as being present in humans, its role in regulating adult metabolism is unclear (85). In mice, BAT contributes to the regulation of body adiposity and thermogenesis. Similar to WAT, brown adipocytes store TAG and, in response 
to locally released norepinephrine, undergo PKA-activated lipolysis. However, brown adipocytes express UCP1, which uncouples the generation of ATP from fat oxidation, resulting in the generation of heat. Fatty acids released by lipolysis directly activate UCP1. Ablation of the Cidea gene in mice, which is predominately expressed in BAT in mice, results in increased BAT lipolysis, temperature, metabolic rate, and a reduction in respiratory exchange ratio consistent with increased fat oxidation (62). Additionally, the presence of Cidea promotes degradation of the AMPK $\beta$ subunit (86), reducing AMPK activation, which may also contribute to reduced oxidative metabolism and protection against diet-induced obesity. Brown adipocytes also express Plin 1. In recent studies using lines of transgenic and Plin1-knockout mice, we demonstrated that the mutation of the six consensus PKA sites of Plin1 from serine to alanine blocked catecholamine-stimulated lipolysis and brown fat thermogenesis (72). Thus, in adult mice, LD proteins regulate BAT metabolism and thermogenesis. Future studies in humans are needed to determine whether there is a similar role of these proteins in human BAT metabolism.

In addition to the PLIN and CIDE proteins, mutations in two additional proteins involved in LD physiology have been linked to the development of human lipodystrophy. The protein seipin has been identified as necessary for maintenance of LD morphology and size (87); mutations in this gene in humans result in a dramatically reduced ability to store TAG within adipocytes (but not in liver or muscle), leading to a generalized lipodystrophy (Berardinelli-Seip congenital lipodystrophy type 2 ) with a near complete absence of adipose tissue from birth associated with acromegaloid features, insulin resistance, diabetes, hypertriglyceridemia, and hepatic steatosis (88). Similarly, caveolin-1, which is a component of caveolae, specialized membrane invaginations, is required for $\mathrm{LD}$ formation and is found as part of the LD proteome (89-91). One patient has been identified with a homozygous nonsense mutation of caveolin-1 that resulted in the clinical features of Berardinelli-Seip congenital lipodystrophy (92). To summarize, reduced expression in several different LD proteins has been linked to the development of lipoatrophy in mice and humans, which leads to insulin resistance and metabolic dysfunction.

\section{Role of CIDE/PLIN1 in obese animals and humans}

Obese individuals have reduced expression of CIDEA in their adipocytes, which appears to correlate with features of the metabolic syndrome (65). Interestingly, knockdown of CIDEA in human adipocytes increases both lipolysis and the expression of TNF- $\alpha$, a cytokine implicated in the development of obesity-associated insulin resistance. One study reported that PLIN1 mRNA and protein expression was reduced in adipocytes in morbidly obese men and women (93), although another laboratory found that PLIN1 expression increases with obesity (94). Other investigators have linked levels of PLIN1 expression in adipocytes to rates of constitutive lipolysis (95), an observation that is consistent with a reduction of PLIN1 with obesity. Obese animals have also been found to have dramatically reduced expression of Plin1 (96). A recent study investigated whether expression of PLIN1, CIDEA, and CIDEC differed in obese individuals based on insulin sensitivity. Importantly, the expression of PLIN1, CIDEA, and CIDEC is reduced in both subcutaneous and omental adipose tissue of insulin-resistant individuals compared with weight-matched insulin sensitive individuals (57). Presumably, the individuals with reduced expression of these LD proteins had increased adipocyte lipolysis that resulted in increased levels of circulating fatty acids and insulin resistance (Figure 1). Particularly relevant in this regard, as we noted previously, TNF- $\alpha$ reduces expression of PLIN1 $(68,97)$ and CIDEC $(75,76)$ in adipocytes, while enhancing PKA activation in human adipocytes (98) and increasing fatty acid release. Treatment with thiazolidinediones blocks TNF- $\alpha$-induced lipolysis in part by blocking the decrease in Plin1 expression (68). Of further relevance to these observations is that fatty acids can activate inflammatory pathways by engaging TLRs on cells. Increased rates of lipolysis have been linked to recruitment of macrophages in adipose tissue (refs. 99, 100; Figure 1). Together these observations provide a mechanistic framework linking two hallmarks of obesity that are implicated in the development of obesity complications, i.e., adipose tissue inflammation and fatty acid flux.

Several studies have found that polymorphisms in the PLIN1 (101-107) and CIDE $(108,109)$ genes influence body weight and the risk of metabolic disease. Interestingly, one PLIN1 polymorphism was found to be associated with reduced PLIN1 expression and increased rates of basal and stimulated adipocyte lipolysis (95). Consistent with the increased rates of lipolysis, humans with this polymorphism tend to have reduced body weight and body fat mass $(106,110)$. PLIN1 polymorphisms have also been suggested to affect the ability to lose weight on a diet $(101,103)$, as well as the metabolic response to exercise (111). More work is needed to confirm and extend these observations among different human populations.

\section{Role of LDs in non-alcoholic fatty liver disease and nonalcoholic steatohepatitis}

In obese humans, nonalcoholic fatty liver disease (NAFLD) is becoming more prevalent $(112,113)$ and can lead to nonalcoholic steatohepatitis (NASH), cirrhosis, and even cancer (114). Hepatic steatosis is often associated with resistance to the actions of insulin on hepatic gluconeogenesis, while hepatocytes remain sensitive to the lipogenic actions of insulin (115). In obesity, fatty acids released from adipocytes are an important source of fatty acids that are found as TAG within hepatic LDs (116). There are a limited number of papers that have investigated the role of LD proteins in human liver, with the greatest number focusing on the PAT proteins. In normal liver both PLIN2 and PLIN3 have been found to be associated with LDs (46). However, with the development of hepatic steatosis, PLIN1 protein is found localized to hepatocyte LDs (46). Consistent with this histologic observation, two separate studies, which used microarray analysis to determine the expression pattern of proteins in NAFLD, revealed PLIN1 as one of the most prominent upregulated genes $(117,118)$. Interestingly, in steatotic hepatocytes, the pattern of PLIN1, -2 , and -3 proteins differ depending upon the size of the LDs and their intracellular localization (46). In a second study, the association of PLIN1 and PLIN2 with LDs were observed in subjects with both NAFLD and NASH (119). PLIN1 tended to be associated with larger LDs, while expression of PLIN2 was more correlated with the presence of ballooned hepatocytes and evidence of oxidation (119). The possible significance and differing roles of PLIN1 and -2 in liver disease is an important unanswered question.

The liver contains several lipases, including ATGL, HSL, TAG lipase (120), and a related lipase called "PNLPA3" (ref. 121; Figure 2). In a genome-wide scan, mutations in PNPLA3 were associated with an increased risk of NAFLD in Hispanics and reduced risk in African Americans (121). PNPLA3 was identified as a LD protein that hydrolyzes TAG (122). More recently a missense mutation 


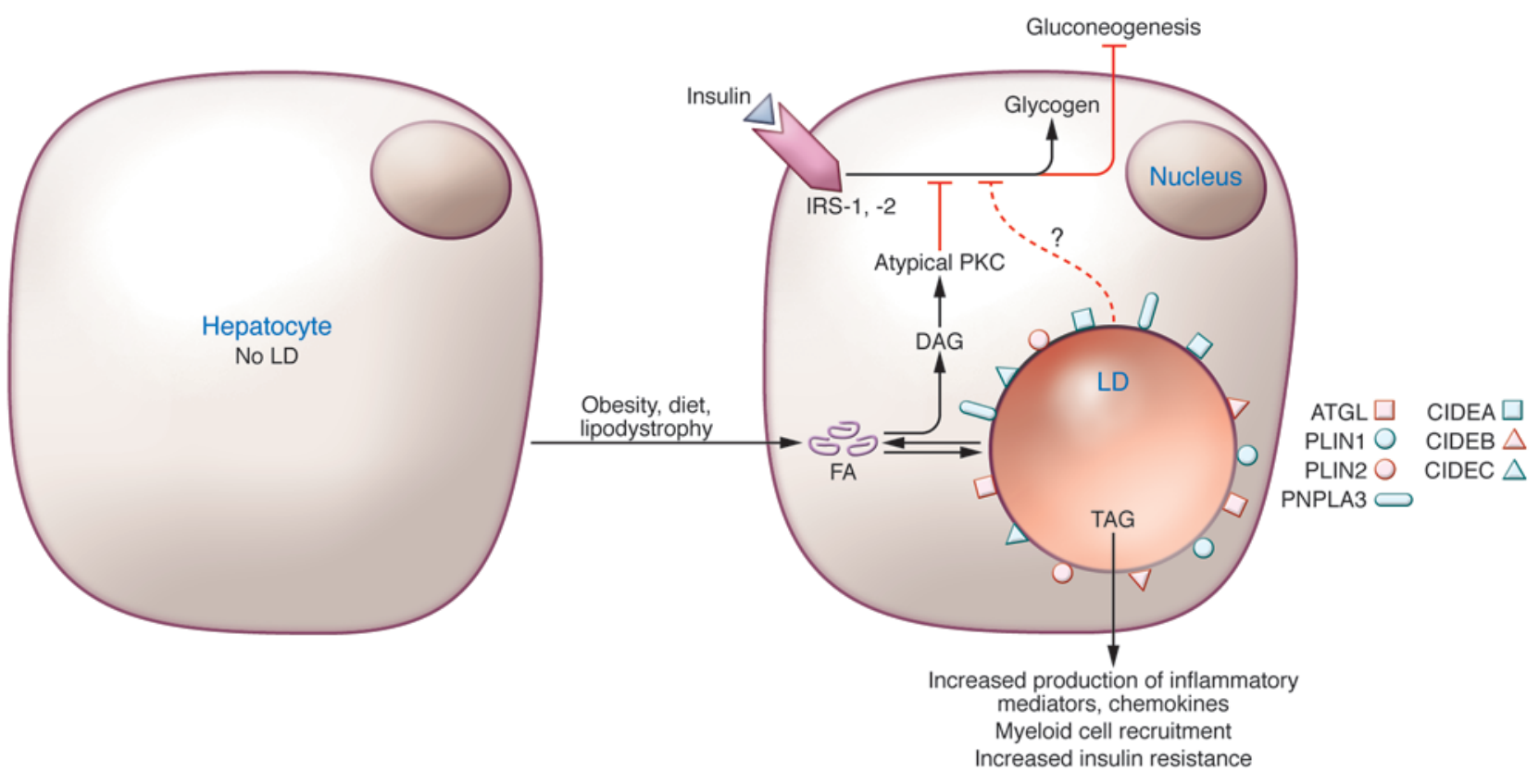

\section{Figure 2}

Liver LD increases in obesity are linked to hepatic steatosis and insulin resistance. Fatty acids from the diet or adipocyte lipolysis result in accumulation of neutral lipids in LD in hepatocytes and incorporation of PLIN2, PLIN3, CIDEA, CIDEB, CIDEC, and ATGL on the LD surface. In humans, PLIN1 is also found on hepatic LDs in individuals with NAFLD and NASH. Increased fatty acid accumulation and LD formation are in general associated with increased accumulation of diacylglycerol (DAG) and inflammatory cytokines. DAG activates atypical PKC, and fatty acids and cytokines activate inflammatory signaling pathways, which cumulatively block the actions of IRS-1 and -2 and promote insulin resistance. It is possible that DAG as well as TAG accumulate in LDs. In hepatocytes, insulin resistance is marked by increased hepatic gluconeogenesis and reduced glycogen formation. Notably, mutations in the phospholipase PNPLA3 result in hepatic steatosis.

(L148M) in PNPLA3 was associated with increased risk of developing NAFLD. Importantly, in vitro studies revealed that this mutation in PNPLA3 ablated the ability of the protein to hydrolyze TAG (122). However, it is important to note that PNPLA3-knockout mice do not develop hepatic steatosis (123), which suggests that the PNPLA3 mutants identified in humans might interfere with other proteins involved in normal hepatic LD metabolism.

While we have limited knowledge of how PLIN2 accumulation regulates the accumulation of TAG in hepatocytes of humans, most of our knowledge about the in vivo role of these LD proteins is inferred from studies in rodents. Plin2 and Plin3, but not Plin1, are generally associated with hepatic LDs of rodents. More recently we found that addition of alcohol to a high-fat diet resulted in expression of both Plin 1 and -2 and hepatocyte LD (124), but the importance and mechanisms that result in Plin 1 expression here are unclear. Loss of Plin2 expression in mice reduced the effect of high-fat diet-induced liver TAG accumulation (125). When the Plin2-knockout mice were crossed with $o b / o b$ mice, the insulin resistance of the double-knockout mice improved in both the liver and skeletal muscle (126). In ob/ob/Plin2-null mice, liver fat accumulation was reduced by $25 \%$, perhaps secondary to increased VLDL secretion. With loss of Plin2, Plin3 became associated with the LDs, while Plin5 expression was greatly increased. Knockout or knockdown of Plin2 in the liver reduces hepatic TAG levels in both genetic and diet-induced obese mice and improves hepatic insulin sensitivity (125-128). The increased insulin sensitivity in mice with reduced or ablated Plin2 might be explained by reduced hepatic diacylglycerol content $(128,129)$.
Previous studies had suggested that one mechanism by which Plin2 expression reduces lipid hydrolysis was that it excludes ATGL from the droplet (130). In support of this observation, overexpression of Plin2 significantly decreases TAG hydrolysis in hepatocytes, while siRNA knockdown of Plin2 or overexpression of ATGL enhances TAG hydrolysis (131). Interestingly, altering TAG hydrolysis through manipulations of Plin2 or ATGL regulates PPAR $\alpha$ activity and target gene expression. Specifically, ATGL overexpression and Plin 2 knockdown activate PPAR $\alpha$, whereas Plin2 overexpression inhibits PPAR $\alpha$ activity and fat oxidation $(128,131)$. Plin2 ablation may reduce hepatic TAG and increase insulin sensitivity in obese mice by several mechanisms. In contrast, combined knockdown of both Plin 2 and Plin 3 in cultured hepatocytes exposed to fatty acids results in significantly increased insulin resistance (132). In summary, reduction of Plin2 promotes a reduction in hepatic steatosis and increases insulin sensitivity, but a reduction in both Plin 2 and Plin 3 causes insulin resistance.

The CIDE proteins are also expressed on hepatocyte LDs when the liver accumulates TAG, such as in $o b / o b$ mice (6). In hepatocytes, the transcription factor PPAR $\gamma$ increases Cidec expression (6), and SREBP1 regulates Cidea (133). Adenovirus-mediated overexpression of Cidec in liver increases TAG accumulation in lean mice, whereas knockdown of FSP27 in ob/ob mice reduces hepatic TAG accumulation (6). In mice, Cideb is predominately expressed in liver, less expressed in kidney, and expressed very little in small intestine and colon. Cideb is thought to be associated both with the endoplasmic reticulum and LDs (61). Cideb-knockout mice are protected against diet-induced obesity, have increased fat oxidation, and are 
insulin sensitive (134). Cideb within the liver is necessary for the acquisition of TAG and apoB binding in the developing VLDL particle (61). Thus the CIDE proteins have an important but not completely understood role in hepatic LD metabolism.

\section{LDs and skeletal muscle metabolism}

Our understanding of the role of LDs in regulating metabolism in skeletal muscle is less well developed, and a function of PAT proteins in regulating insulin action in skeletal muscle of obese and diabetic individuals has not been well delineated. Studies conflict as to whether the level of PLIN2, or possibly PLIN5, is related to insulin resistance in obese, diabetic individuals $(135,136)$. Tissue-specific ablation of Plin 2 and -5 in skeletal muscle will provide important insights into their actions. In certain states, skeletal muscle can store increased amounts of TAG in LDs, which correlates with insulin resistance (137). Intriguingly, SNARE proteins such as SNAP23 are required for both LD fusion (138) as well as insulin-mediated vesicular trafficking of Glut4 to the plasma membrane. When muscle cells were incubated with oleic acid, a complement of SNAP23 proteins appeared to be diverted from the plasma membrane to the surface of the newly formed LDs (138). SNAP23 sequestration on LDs of oleic acid-treated cells was coincident with attenuated insulin-stimulated glucose transport. Moreover, consistent with these in vitro observations, more SNAP23 was localized to the non-plasma membrane pool in muscle cells of type 2 diabetics in whom intracellular lipid was increased (139).

Mutations in genes involved in LD turnover have been linked to lipid storage disease and myopathy in humans. Mutations in ATGL result in a form of neutral lipid storage disease with myopathy that is manifest by TAG accumulation in skeletal and cardiac muscle, liver, and leukocytes (140-142). Other than myopathy, metabolic manifestations have been variable, with most patients having normal glucose and lipid levels (140-142). Mutations in CGI-58 also result in neutral lipid storage disease (Chanarin-Dorfman syndrome) and are associated with ichthyosis $(143,144)$. Thus, there are TAG droplets observed in skin, liver, and leukocytes. No mutations have yet been described in other genes involved in LD hydrolysis, such as HSL.

One complicating issue in relating muscle TAG to insulin sensitivity is that the muscle of elite athletes contains high amounts of myocellular fat in the absence of insulin resistance (145). A possible explanation for this paradox may lie in the fact that exercise increases the expression of the enzyme DGAT1 in skeletal muscle (146). DGAT1 catalyzes the addition of a fatty acyl group to diacylglycerol to form TAG. Transgenic mice overexpressing Dgat 1 in skeletal muscle are protected against the development high-fat diet-induced insulin resistance (147). These Dgat 1 transgenic mice have increased TAG in skeletal muscle, but less diacylglycerol and reduced activation of the

PKC isozymes that promote insulin resistance. Muscles from these transgenic mice also have increased fatty acid oxidation (148).

The observation that DGAT overexpression promotes the formation of TAG without causing insulin resistance has been replicated in other tissues besides muscle. Overexpressing DGAT in liver results in hepatic steatosis but does not cause insulin resistance (149). One line of Dgat1 transgenic mice in which Dgat1 was overexpressed in both adipocytes and macrophages or in macrophages alone was protected against diet-induced insulin resistance $(150,151)$. Furthermore, overexpression of Dgat 1 in heart protects against cardiac lipotoxicity (152). These data underscore the importance to metabolic health of sequestering excess free fatty acids in esterified form (TAG) within LDs.

\section{Conclusion}

All cells can form and sequester neutral lipids within LDs, especially when exposed to increased levels of fatty acids. At present, it is evident that proteins in the PAT and CIDE families can facilitate the storage of fatty acids within LDs and reduce rates of hydrolysis. However, these LD-associated proteins differ in their ability to modulate lipid hydrolysis. Our appreciation of the multiple physiologic and pathophysiologic roles of LDs continues to evolve, with LDs now recognized as important regulators of atherosclerosis $(153,154)$, cancer $(155)$, and viral replication (156). It is exciting that we are just beginning to appreciate the full complexity and importance of LD metabolism. Greater understanding of LD biogenesis, composition, and heterogeneity will provide new insights in understanding the role of LDs in health and disease.

\section{Acknowledgments}

This work was supported by grants from the American Diabetes Association (7-08-RA-57 to A.S. Greenberg; 7-07-JF-43 to D.G. Mashek), the USDA Agricultural Research Service (58-1950-7-707 to A.S. Greenberg), the Department of Veterans Affairs Office of Research and Development Medical Research Service (to F.B. Kraemer) and the NIH (AG028098 to F.B. Kraemer; DK0822574, 1RC2ES018781, and R24DK087669 to A.S. Greenberg; DK56598 and DK59935 to R.A. Coleman; RO1-HD045962 and PO1-HD38129 to J.L. McManaman; 3P30DK046200 to V. Puri; DK074979 to M.S. Obin; and DK085008 to D.G. Mashek). The Obesity Review Series is supported in part by unrestricted educational grants from Merck \& Co. and the Life Sciences Institute of the University of Michigan.

Address correspondence to: Andrew S. Greenberg, Tufts University, 711 Washington Street, Boston, Massachusetts 02111, USA. Phone: 617.556.3144; Fax: 617.556.3224; E-mail: Andrew. greenberg@tufts.edu.

1. Dresner A, et al. Effects of free fatty acids on glucose transport and IRS-1 associated phosphoatidylinositiol 3-kinase activit. J Clin Invest. 1999;103(2):253-259.

2. Boden $\mathrm{G}$, et al. Free fatty acids produce insulin resistance and activate the proinflammatory nuclear factor- $\{$ kappa\}b pathway in rat liver. Diabetes. 2005;54(12):3458-3465.

3. Koutsari C, Jensen MD. Thematic review series: patient-oriented research. Free fatty acid metabolism in human obesity. J Lipid Res. 2006;47(8):1643-1650.

4. Chawla A, et al. PPARdelta is a very low-density lipoprotein sensor in macrophages. Proc Natl Acad Sci US A. 2003;100(3):1268-1273.

5. Edvardsson U, et al. PPARalpha activation increases triglyceride mass and adipose differentiationrelated protein in hepatocytes. J Lipid Res. 2006;
47(2):329-340.

6. Matsusue $\mathrm{K}$, et al. Hepatic steatosis in leptin-deficient mice is promoted by the PPARgamma target gene Fsp27. Cell Metab. 2008;7(4):302-311.

7. Listenberger LL, et al. Triglyceride accumulation protects against fatty acid-induced lipotoxicity. Proc Natl Acad Sci U S A. 2003;100(6):3077-3082.

8. James CN, et al. Disruption of the Arabidopsis CGI-58 homologue produces Chanarin-Dorfmanlike lipid droplet accumulation in plants. Proc Natl Acad Sci U S A. 2010;107(41):17833-17838.

9. Guo Y, Cordes KR, Farese RV Jr, Walther TC. Lipid droplets at a glance. J Cell Sci. 2009;122(Pt 6):749-752.

10. Goodman JM. The gregarious lipid droplet. J Biol Chem. 2008;283(42):28005-28009

11. Murphy D. The biogenesis of lipid bodies in ani- mals, plants, and microorganisms. Prog Lipid Res. 2001;40(5):325-438.

12. Coleman RA, Lee DP. Enzymes of triacylglycerol synthesis and their regulation. Prog Lipid Res. 2004;43(2):134-176.

13. Stone SJ, Levin MC, Zhou P, Han J, Walther TC, Farese RV Jr. The endoplasmic reticulum enzyme DGAT2 is found in mitochondria-associated membranes and has a mitochondrial targeting signal that promotes its association with mitochondria. J Biol Chem. 2009;284(8):5352-5361.

14. Blanchette-Mackie EJ, et al. Perilipin is located on the surface layer of intracellular lipid droplets in adipocytes. J Lipid Res. 1995;36(6):1211-1226.

15. Novikoff AB, Novikoff PM, Rosen OM, Rubin CS. Organelle relationships in cultured 3T3-L1 preadi- 
pocytes. J Cell Biol. 1980;87(1):180-196.

16. Bell RM, Coleman RA. Enzymes of glycerolipid synthesis in eukaryotes. Annu Rev Biochem. 1980;49:459-487.

17. Walther TC, Farese RV Jr. The life of lipid droplets. Biochim Biophys Acta. 2009;1791(6):459-466.

18. Guo Y, et al. Functional genomic screen reveals genes involved in lipid-droplet formation and utilization. Nature. 2008;453(7195):657-661.

19. Beller M, et al. COPI complex is a regulator of lipid homeostasis. PLoS Biol. 2008;6(11):e292.

20. Athenstaedt K, Zweytick D, Jandrositz A, Kohlwein $\mathrm{SD}$, Daum G. Identification and characterization of major lipid particle proteins of the yeast Saccharomyces cerevisiae. J Bacteriol. 1999;181(20):6441-6448.

21. Wu CC, Howell KE, Neville MC, Yates JR 3rd, McManaman JL. Proteomics reveal a link between the endoplasmic reticulum and lipid secretory mechanisms in mammary epithelial cells. Electrophoresis. 2000;21(16):3470-3482.

22. Liu P, Ying Y, Zhao Y, Mundy DI, Zhu M, Anderson RG. Chinese hamster ovary K2 cell lipid droplets appear to be metabolic organelles involved in membrane traffic. J Biol Chem. 2004;279(5):3787-3792.

23. Fujimoto $Y$, et al. Identification of major proteins in the lipid droplet-enriched fraction isolated from the human hepatocyte cell line HuH7. Biochim Biophys Acta. 2004;1644(1):47-59.

24. Umlauf E, Csaszar E, Moertelmaier M, Schuetz GJ, Parton RG, Prohaska R. Association of stomatin with lipid bodies. J Biol Chem. 2004;279(22):23699-23709.

25. Brasaemle DL, Dolios G, Shapiro L, Wang R. Proteomic analysis of proteins associated with lipid droplets of basal and lipolytically stimulated 3T3-L1 adipocytes. J Biol Chem. 2004;279(45):46835-46842.

26. Miura S, et al. Functional conservation for lipid storage droplet association among perilipin, ADRP, and TIP47-related proteins in mammals, drosophila, and dictyostelium. J Biol Chem. 2002; 277(35):32253-32257.

27. Paciga M, McCudden CR, Londos C, DiMattia GE, Wagner GF. Targeting of big stanniocalcin and its receptor to lipid storage droplets of ovarian steroidogenic cells. J Biol Chem. 2003;278(49):49549-49554.

28. Wolins NE, Skinner JR, Schoenfish MJ, Tzekov A, Bensch KG, Bickel PE. Adipocyte protein S312 coats nascent lipid droplets. J Biol Chem. 2003; 278(39):37713-37721.

29. Bartz R, et al. Dynamic activity of lipid droplets: protein phosphorylation and GTP-mediated protein translocation. J Proteome Res. 2007;6(8):3256-3265.

30. Beller M, et al. Characterization of the Drosophila lipid droplet subproteome. Mol Cell Proteomics. 2006;5(6):1082-1094

31. Cermelli S, Guo Y, Gross SP, Welte MA. The lipiddroplet proteome reveals that droplets are a proteinstorage depot. Curr Biol. 2006;16(18):1783-1795.

32. Wan HC, Melo RC, Jin Z, Dvorak AM, Weller PF. Roles and origins of leukocyte lipid bodies: proteomic and ultrastructural studies. FASEB J. 2007;21(1):167-178.

33. Greenberg AS, Egan JJ, Wek SA, Garty NB, Blanchette-Mackie EJ, Londos C. Perilipin, a major hormonally regulated adipocyte-specific phosphoprotein associated with the periphery of lipid storage droplets. J Biol Chem. 1991;266(17):11341-11346.

34. Londos C, Brasaemle D, Schultz C, Segrest J, Kimmel A. Perilipins, ADRP, and other proteins that associate with intracellular neutral lipid droplets in animal cells. Semin Cell Dev Biol. 1999;10(1):51-58.

35. Greenberg AS, Egan JJ, Wek SA, Moos MC Jr, Londos C, Kimmel AR. Isolation of cDNAs for perilipins A and B: sequence and expression of lipid droplet-associated proteins of adipocytes. Proc Natl Acad Sci U S A. 1993;90(24):12035-12039.

36. Lu X, et al. The murine perilipin gene: the lipid droplet-associated perilipins derive from tissue-specific, mRNA splice variants and define a gene of ancient origin. Mammalian Genome. 2001;12(9):741-749.
37. Miura S, et al. Functional conservation for lipid storage droplet association among Perilipin, ADRP, and TIP47 (PAT)-related proteins in mammals, Drosophila, and Dictyostelium. J Biol Chem. 2002; 277(35):32253-32257.

38. Souza S, et al. Modulation of hormone-sensitive lipase and protein kinase-A-mediated lipolysis by perilipin A in an adenoviral reconstituted system. J Biol Chem. 2002;277(10):8267-8272.

39. Brasaemle D, Rubin B, Harten I, Gruia-Gray J, Kimmel A, Londos C. Perilipin A increases triacylglycerol storage by decreasing the rate of triacylglycerol hydrolysis. J Biol Chem. 2000;275(49):38486-38493.

40. Brasaemle D, Barber T, Wolins N, Serrero G, Blanchette-Mackie E, Londos C. Adipose differentiation related protein is an ubiquitously expressed lipid storage droplet-asociated protein. J Lipid Res. 1997;38(11):2249-2263

41. Imamura M, et al. ADRP stimulates lipid accumulation and lipid droplet formation in murine fibroblasts. Am J Physiol Endocrinol Metab. 2002; 283(4):E775-E783.

42. Heid H, Moll R, Schwetlick I, Rackwitz H-R, Keenan T. Adipophilin is a specific marker of lipid accumulation in diverse cell types and diseases. Cell Tissue Research. 1998;294(2):309-321.

43. Wolins N, Rubin B, Brasamele D. TIP47 associates with lipid droplets. J Biol Chem. 2001; 276(7):5101-5108.

44. Wolins NE, et al. OXPAT/PAT-1 is a PPAR-induced lipid droplet protein that promotes fatty acid utilization. Diabetes. 2006;55(12):3418-3428.

45. Wolins NE, Quaynor BK, Skinner JR, Schoenfish MJ, Tzekov A, Bickel PE. S3-12, Adipophilin, and TIP47 package lipid in adipocytes. J Biol Chem. 2005; 280(19):19146-19155.

46. Straub BK, Stoeffel P, Heid H, Zimbelmann R, Schirmacher P. Differential pattern of lipid dropletassociated proteins and de novo perilipin expression in hepatocyte steatogenesis. Hepatology. 2008; 47(6):1936-1946.

47. Lass A, Zimmermann R, Oberer M, Zechner R Lipolysis - a highly regulated multi-enzyme complex mediates the catabolism of cellular fat stores. Prog Lipid Res. 2011;50(1):14-27.

48. Yang $X$, et al. The $G(0) / G(1)$ switch gene 2 regulates adipose lipolysis through association with adipose triglyceride lipase. Cell Metab. 2010;11(3):194-205.

49. Lass A, et al. Adipose triglyceride lipase-mediated lipolysis of cellular fat stores is activated by CGI-58 and defective in Chanarin-Dorfman Syndrome. Cell Metab. 2006;3(5):309-319.

50. Egan J, et al. Mechanism of hormone-stimulated lipolysis in adipocytes: translocation of hormonesensitive lipase to the lipid storage droplet. Proc Natl Acad Sci, USA. 1992;89(18):8537-8541.

51. Miyoshi $\mathrm{H}$, et al. Perilipin promotes hormone-sensitive lipase-mediated adipocyte lipolysis via phosphorylation-dependent and -independent mechanisms. J Biol Chem. 2006;281(23):15837-15844.

52. Subramanian V, et al. Perilipin A mediates the reversible binding of CGI-58 to lipid droplets in 3T3-L1 adipocytes. J Biol Chem. 2004;279(40):42062-42071.

53. Granneman JG, Moore HP, Granneman RL, Greenberg AS, Obin MS, Zhu Z. Analysis of lipolytic protein trafficking and interactions in adipocytes. J Biol Chem. 2007;282(8):5726-5735.

54. Miyoshi H, Perfield JW 2nd, Obin MS, Greenberg AS. Adipose triglyceride lipase regulates basal lipolysis and lipid droplet size in adipocytes. $J$ Cell Biochem. 2008;105(6):1430-1436.

55. Haemmerle G, et al. Defective lipolysis and altered energy metabolism in mice lacking adipose triglyceride lipase. Science. 2006;312(5774):734-737.

56. Liang L, Zhao M, Xu Z, Yokoyama KK, Li T. Molecular cloning and characterization of CIDE-3, a novel member of the cell-death-inducing DNAfragmentation-factor (DFF45)-like effector family.
Biochem J. 2003;370(pt 1):195-203.

57. Puri V, et al. Cidea is associated with lipid droplets and insulin sensitivity in humans. Proc Natl Acad Sci US A. 2008;105(22):7833-7838.

58. Puri V, et al. Fat-specific protein 27, a novel lipid droplet protein that enhances triglyceride storage. J Biol Chem. 2007;282(47):34213-34218.

59. Keller P, et al. Fat-specific protein 27 regulates storage of triacylglycerol. J Biol Chem. 2008; 283(21):14355-14365.

60. Liu K, et al. Functional analysis of FSP27 protein regions for lipid droplet localization, caspase-dependent apoptosis, and dimerization with CIDEA. Am J Physiol Endocrinol Metab. 2009;297(6):E1395-E1413.

61. Ye J, et al. Cideb, an ER- and lipid droplet-associated protein, mediates VLDL lipidation and maturation by interacting with apolipoprotein B. Cell Metab. 2009;9(2):177-190.

62. Zhou $Z$, et al. Cidea-deficient mice have lean phenotype and are resistant to obesity. Nat Genet. 2003; 35(1):49-56.

63. Nishino N, et al. FSP27 contributes to efficient energy storage in murine white adipocytes by promoting the formation of unilocular lipid droplets. J Clin Invest. 2008;118(8):2808-2821.

64. Toh SY, et al. Up-regulation of mitochondrial activity and acquirement of brown adipose tissue-like property in the white adipose tissue of $\mathrm{fsp} 27$ deficient mice. PLoS One. 2008;3(8):e2890.

65. Nordstrom EA, et al. A human-specific role of cell death-inducing DFFA (DNA fragmentation factoralpha)-like effector A (CIDEA) in adipocyte lipolysis and obesity. Diabetes. 2005;54(6):1726-1734

66. Coppack SW, Jensen MD, Miles JM. In vivo regulation of lipolysis in humans. J Lipid Res. 1994; 35(2):177-193.

67. Boden G. Role of fatty acids in the pathogenesis of insulin resistance and NIDDM. Diabetes. 1997; 46(1):3-10.

68. Souza SC, Yamamoto M, Franciosa M, Lien P, Greenberg A. BRL blocks the lipolytic actions of tumor necrosis factor-alpha (TNF-a): A Potential New Insulin-Sensitizing Mechanism for the Thiazolidinediones. Diabetes. 1998;47(4):691-695.

69. Souza SC, et al. Overexpression of perilipin A and B blocks the ability of tumor necrosis factor alpha to increase lipolysis in 3T3-L1 adipocytes. J Biol Chem. 1998;273(38):24665-24669.

70. Tansey JT, et al. Perilipin ablation results in a lean mouse with aberrant adipocyte lipolysis, enhanced leptin production, and resistance to diet-induced obesity. Proc Natl Acad Sci U S A. 2001;98(11):6494-6499.

71. Martinez-Botas J, et al. Absence of perilipin results in leanness and reverses obesity in Leprdb/db mice. Nat Genet. 2000;26(4):474-479.

72. Souza SC, et al. Perilipin regulates the thermogenic actions of norepinephrine in brown adipose tissue. J Lipid Res. 2007;48(6):1273-1279.

73. Kovsan J, Ben-Romano R, Souza SC, Greenberg AS, Rudich A. Regulation of adipocyte lipolysis by degradation of the perilipin protein: nelfinavir enhances lysosome-mediated perilipin proteolysis. J Biol Chem. 2007;282(30):21704-21711.

74. Gandotra S, et al. Perilipin deficiency and autosomal dominant partial lipodystrophy. N Engl J Med. 2011;364(8):740-748.

75. Ranjit S, et al. Regulation of Fat Specific Protein 27 by Isoproterenol and TNF-\{alpha\} to control lipolysis in murine adipocytes. J Lipid Res. 2011; 52(2):221-236.

76. Kim JY, Liu K, Zhou S, Tillison K, Wu Y, Smas CM. Assessment of fat-specific protein 27 in the adipocyte lineage suggests a dual role for FSP27 in adipocyte metabolism and cell death. Am J Physiol Endocrinol Metab. 2008;294(4):E654-E667.

77. Rubio-Cabezas O, et al. Partial lipodystrophy and insulin resistant diabetes in a patient with a homozygous nonsense mutation in CIDEC. EMBO Mol 
Med. 2009;1(5):280-287.

78. Granneman JG, Li P, Zhu Z, Lu Y. Metabolic and cellular plasticity in white adipose tissue I: effects of beta3-adrenergic receptor activation. Am J Physiol Endocrinol Metab. 2005;289(4):E608-E616.

79. Li P, Zhu Z, Lu Y, Granneman JG. Metabolic and cellular plasticity in white adipose tissue II: role of peroxisome proliferator-activated receptor-alpha. AmJ Physiol Endocrinol Metab. 2005;289(4):E617-E626.

80. Gauthier MS, et al. AMP-activated protein kinase is activated as a consequence of lipolysis in the adipocyte: potential mechanism and physiological relevance. J Biol Chem. 2008;283(24):16514-16524.

81. Castro-Chavez F, et al. Coordinated upregulation of oxidative pathways and downregulation of lipid biosynthesis underlie obesity resistance in perilipin knockout mice: a microarray gene expression profile. Diabetes. 2003;52(11):2666-2674.

82. Saha PK, Kojima H, Martinez-Botas J, Sunehag AL, Chan L. Metabolic adaptations in the absence of perilipin: increased beta-oxidation and decreased hepatic glucose production associated with peripheral insulin resistance but normal glucose tolerance in perilipin-null mice. J Biol Chem. 2004; 279(34):35150-35158.

83. Miyoshi $\mathrm{H}$, et al. Perilipin overexpression in mice protects against diet-induced obesity. J Lipid Res. 2010;51(5):975-982.

84. Sawada T, et al. Perilipin overexpression in white adipose tissue induces a brown fat-like phenotype. PLoS One. 2010;5(11):e14006.

85. Cypess AM, Kahn CR. The role and importance of brown adipose tissue in energy homeostasis. Curr Opin Pediatr. 2010;22(4):478-484.

86. Qi J, et al. Downregulation of AMP-activated protein kinase by Cidea-mediated ubiquitination and degradation in brown adipose tissue. EMBOJ. 2008; 27(11):1537-1548

87. Szymanski KM, et al. The lipodystrophy protein seipin is found at endoplasmic reticulum lipid droplet junctions and is important for droplet morphology. Proc Natl Acad Sci U S A. 2007;104(52):20890-20895.

88. Magre J, et al. Identification of the gene altered in Berardinelli-Seip congenital lipodystrophy on chromosome 11q13. Nat Genet. 2001;28(4):365-370.

89. Cohen AW, et al. Role of caveolin-1 in the modulation of lipolysis and lipid droplet formation. Diabetes. 2004;53(5):1261-1270.

90. Robenek MJ, et al. Lipids partition caveolin-1 from ER membranes into lipid droplets: updating the model of lipid droplet biogenesis. FASEB J. 2004; 18(7):866-868

91. Blouin CM, Le Lay S, Lasnier F, Dugail I, Hajduch E. Regulated association of caveolins to lipid droplets during differentiation of 3T3-L1 adipocytes. Biochem Biophys Res Commun. 2008;376(2):331-335.

92. Kim CA, et al. Association of a homozygous nonsense caveolin-1 mutation with Berardinelli-Seip congenital lipodystrophy. J Clin Endocrinol Metab. 2008;93(4):1129-1134.

93. Wang Y, et al. Perilipin expression in human adipose tissues: effects of severe obesity, gender, and depot. Obes Res. 2003;11(8):930-936.

94. Kern PA, Di Gregorio G, Lu T, Rassouli N, Ranganathan G. Perilipin Expression in Human Adipose Tissue Is Elevated with Obesity. J Clin Endocrinol Metab. 2004;89(3):1352-1358.

95. Mottagui-Tabar S, et al. Evidence for an important role of perilipin in the regulation of human adipocyte lipolysis. Diabetologia. 2003;46(6):789-797.

96. Gaidhu MP, Anthony NM, Patel P, Hawke TJ, Ceddia RB. Dysregulation of lipolysis and lipid metabolism in visceral and subcutaneous adipocytes by high-fat diet: role of ATGL, HSL, and AMPK. AmJ Physiol Cell Physiol. 2010;298(4):C961-C971.

97. Ryden M, Arvidsson E, Blomqvist L, Perbeck L, Dicker A, Arner P. Targets for TNF-alpha-induced lipolysis in human adipocytes. Biochem Biophys Res
Commun. 2004;318(1):168-175.

98. Zhang H, Halbleib M, Ahmed F, Manganiello V, Greenberg A. Tumor necrosis factor-alpha stimulates lipolysis in differentiated human adipocytes through activation of extracellular signal related kinase and elevated extracelular related kinase and elevation of intracellular cAMP. Diabetes. 2002;51(10):2929-2935.

99. Mottillo EP, Shen XJ, Granneman JG. beta3-adrenergic receptor induction of adipocyte inflammation requires lipolytic activation of stress kinases p38 and JNK. Biochim Biophys Acta. 2010;1801(9):1048-1055.

100. Kosteli A, et al. Weight loss and lipolysis promote a dynamic immune response in murine adipose tissue. J Clin Invest. 2010;120(10):3466-3479.

101. Corella D, et al. Obese subjects carrying the $11482 \mathrm{G}>\mathrm{A}$ polymorphism at the perilipin locus are resistant to weight loss after dietary energy restriction. J Clin Endocrinol Metab. 2005;90(9):5121-5126.

102. Sone Y, et al. Association of lifestyle factors, polymorphisms in adiponectin, perilipin and hormone sensitive lipase, and clinical markers in Japanese males. J Nutr Sci Vitaminol (Tokyo). 2010;56(2):123-131.

103. Deram S, et al. Effects of perilipin (PLIN) gene variation on metabolic syndrome risk and weight loss in obese children and adolescents. J Clin Endocrinol Metab. 2008;93(12):4933-4940.

104. Qi L, Zhang C, Greenberg A, Hu FB. Common variations in perilipin gene, central obesity, and risk of type 2 diabetes in US women. Obesity (Silver Spring). 2008;16(5):1061-1065.

105.Qi L, et al. Intragenic linkage disequilibrium structure of the human perilipin gene (PLIN) and haplotype association with increased obesity risk in a multiethnic Asian population. J Mol Med. 2005; 83(6):448-456.

106. Soenen S, et al. Relationship between perilipin gene polymorphisms and body weight and body composition during weight loss and weight maintenance. Physiol Behav. 2009;96(4-5):723-728.

107. Kawai $\mathrm{T}$, et al. Variation in the perilipin gene (PLIN) affects glucose and lipid metabolism in non-Hispanic white women with and without polycystic ovary syndrome. Diabetes Res Clin Pract. 2009; 86(3):186-192.

108.Zhang L, Miyaki K, Nakayama T, Muramatsu M. Cell death-inducing DNA fragmentation factor alphalike effector A (CIDEA) gene V115F (G-->T) polymorphism is associated with phenotypes of metabolic syndrome in Japanese men. Metabolism. 2008; 57(4):502-505.

109.Zhang L, et al. Association of the cell death-inducing DNA fragmentation factor alpha-like effector A (CIDEA) gene V115F (G/T) polymorphism with phenotypes of metabolic syndrome in a Chinese population. Diabetes Res Clin Pract. 2011;91(2):233-238.

110. Qi L, et al. Genetic variation at the perilipin (PLIN) locus is associated with obesity-related phenotypes in White women. Clin Genet. 2004;66(4):299-310.

111.Jenkins NT, McKenzie JA, Damcott CM, Witkowski $\mathrm{S}$, Hagberg JM. Endurance exercise training effects on body fatness, VO2max, HDL-C subfractions, and glucose tolerance are influenced by a PLIN haplotype in older Caucasians. J Appl Physiol. 2010; 108(3):498-506.

112. Szczepaniak LS, et al. Magnetic resonance spectroscopy to measure hepatic triglyceride content: prevalence of hepatic steatosis in the general population. AmJ Physiol Endocrinol Metab. 2005;288(2):E462-E468.

113. Browning JD, et al. Prevalence of hepatic steatosis in an urban population in the United States: impact of ethnicity. Hepatology. 2004;40(6):1387-1395.

114.Hill-Baskin AE, et al. Diet-induced hepatocellular carcinoma in genetically predisposed mice. Hum Mol Genet. 2009;18(16):2975-2988.

115. Brown MS, Goldstein JL. Selective versus total insulin resistance: a pathogenic paradox. Cell Metab. 2008;7(2):95-96.

116.Donnelly KL, Smith CI, Schwarzenberg SJ, Jessurun
J, Boldt MD, Parks EJ. Sources of fatty acids stored in liver and secreted via lipoproteins in patients with nonalcoholic fatty liver disease. J Clin Invest. 2005; 115(5):1343-1351.

117. Greco D, et al. Gene expression in human NAFLD. Am J Physiol Gastrointest Liver Physiol. 2008; 294(5):G1281-G1287.

118. Westerbacka J, et al. Genes involved in fatty acid partitioning and binding, lipolysis, monocyte/ macrophage recruitment, and inflammation are overexpressed in the human fatty liver of insulinresistant subjects. Diabetes. 2007;56(11):2759-2765.

119.Fujii H, et al. Expression of perilipin and adipophilin in nonalcoholic fatty liver disease; relevance to oxidative injury and hepatocyte ballooning. J Atheroscler Thromb. 2009;16(6):893-901.

120. Wei E, et al. Loss of TGH/Ces 3 in mice decreases blood lipids, improves glucose tolerance, and increases energy expenditure. Cell Metab. 2010;11(3):183-193.

121. Romeo S, et al. Genetic variation in PNPLA3 confers susceptibility to nonalcoholic fatty liver disease. Nat Genet. 2008;40(12):1461-1465.

122. He S, et al. A sequence variation (I148M) in PNPLA3 associated with nonalcoholic fatty liver disease disrupts triglyceride hydrolysis. J Biol Chem. 2010; 285(9):6706-6715.

123. Basantani MK, et al. Pnpla3/Adiponutrin deficiency in mice does not contribute to fatty liver disease or metabolic syndrome. J Lipid Res. 2011;52(2):318-329.

124. Orlicky D, et al. Chronic ethanol consumption in mice alters hepatocyte lipid droplet properties [published online ahead of print April 27, 2011]. Alcohol Clin Exp Res. doi:10.1111/j.15300277.2011.01434.x.

125. Chang BH, et al. Protection against fatty liver but normal adipogenesis in mice lacking adipose differentiation-related protein. Mol Cell Biol. 2006; 26(3):1063-1076.

126. Chang BH, Li L, Saha P, Chan L. Absence of adipose differentiation related protein upregulates hepatic VLDL secretion, relieves hepatosteatosis, and improves whole body insulin resistance in leptindeficient mice. J Lipid Res. 2010;51(8):2132-2142.

127. Imai Y, Varela GM, Jackson MB, Graham MJ, Crooke RM, Ahima RS. Reduction of hepatosteatosis and lipid levels by an adipose differentiationrelated protein antisense oligonucleotide. Gastroenterology. 2007;132(5):1947-1954

128.Varela GM, et al. Inhibition of ADRP prevents dietinduced insulin resistance. Am J Physiol Gastrointest Liver Physiol. 2008;295(3):G621-G628.

129.Erion DM, Shulman GI. Diacylglycerol-mediated insulin resistance. Nat Med. 2010;16(4):400-402.

130.Listenberger LL, Ostermeyer-Fay AG, Goldberg EB, Brown WJ, Brown DA. Adipocyte differentiationrelated protein reduces the lipid droplet association of adipose triglyceride lipase and slows triacylglycerol turnover. J Lipid Res. 2007;48(12):2751-2761.

131.Sapiro JM, Mashek MT, Greenberg AS, Mashek DG. Hepatic triacylglycerol hydrolysis regulates peroxisome proliferator-activated receptor alpha activity. J Lipid Res. 2009;50(8):1621-1629.

132. Bell M, et al. Consequences of lipid droplet coat protein downregulation in liver cells: abnormal lipid droplet metabolism and induction of insulin resistance. Diabetes. 2008;57(8):2037-2045.

133. Hall AM, et al. Dynamic and differential regulation of proteins that coat lipid droplets in fatty liver dystrophic mice. J Lipid Res. 2010;51(3):554-563.

134.Li JZ, et al. Cideb regulates diet-induced obesity, liver steatosis, and insulin sensitivity by controlling lipogenesis and fatty acid oxidation. Diabetes. 2007;56(10):2523-2532.

135. Phillips SA, et al. Adipocyte differentiation-related protein in human skeletal muscle: relationship to insulin sensitivity. Obes Res. 2005;13(8):1321-1329.

136. Minnaard R, et al. Adipocyte differentiation-related protein and OXPAT in rat and human skeletal 
muscle: involvement in lipid accumulation and type 2 diabetes mellitus. J Clin Endocrinol Metab. 2009;94(10):4077-4085.

137. Sinha R, et al. Assessment of skeletal muscle triglyceride content by (1) $\mathrm{H}$ nuclear magnetic resonance spectroscopy in lean and obese adolescents: relationships to insulin sensitivity, total body fat, and central adiposity. Diabetes. 2002;51(4):1022-1027.

138. Bostrom P, et al. SNARE proteins mediate fusion between cytosolic lipid droplets and are implicated in insulin sensitivity. Nat Cell Biol. 2007; 9(11):1286-1293

139. Bostrom P, et al. The SNARE protein SNAP23 and the SNARE-interacting protein Munc18c in human skeletal muscle are implicated in insulin resistance/ type 2 diabetes. Diabetes. 2010;59(8):1870-1878.

140 . Fischer $J$, et al. The gene encoding adipose triglyceride lipase (PNPLA2) is mutated in neutral lipid storage disease with myopathy. Nat Genet. 2007; 39(1):28-30.

141.Kobayashi K, et al. The lack of the C-terminal domain of adipose triglyceride lipase causes neutral lipid storage disease through impaired interactions with lipid droplets. J Clin Endocrinol Metab. 2008; 93(7):2877-2884

142.Akiyama M, Sakai K, Ogawa M, McMillan JR, Sawamura D, Shimizu H. Novel duplication mutation in the patatin domain of adipose triglyceride lipase (PNPLA2) in neutral lipid storage disease with severe myopathy. Muscle Nerve. 2007;36(6):856-859.

143. Schweiger M, Lass A, Zimmermann R, Eichmann TO, Zechner R. Neutral lipid storage disease: genetic disorders caused by mutations in adipose triglyceride lipase/PNPLA2 or CGI-58/ABHD5. Am J Physiol Endocrinol Metab. 2009;297(2):E289-E296.

144.Yamaguchi T, Osumi T. Chanarin-Dorfman syndrome: deficiency in CGI-58, a lipid droplet-bound coactivator of lipase. Biochim Biophys Acta. 2009; 1791(6):519-523.

145. Meex RC, et al. Restoration of muscle mitochondrial function and metabolic flexibility in type 2 diabetes by exercise training is paralleled by increased myocellular fat storage and improved insulin sensitivity. Diabetes. 2010;59(3):572-579.

146. Ikeda S, et al. Up-regulation of SREBP-1c and lipogenic genes in skeletal muscles after exercise training. Biochem Biophys Res Commun. 2002;296(2):395-400.

147. Liu L, Zhang Y, Chen N, Shi X, Tsang B, Yu YH Upregulation of myocellular DGAT1 augments triglyceride synthesis in skeletal muscle and protects against fat-induced insulin resistance. J Clin Invest. 2007;117(6):1679-1689.

148. Liu L, et al. Paradoxical coupling of triglyceride synthesis and fatty acid oxidation in skeletal muscle overexpressing DGAT1. Diabetes. 2009;58(11):2516-2524.

149. Monetti M, et al. Dissociation of hepatic steato- sis and insulin resistance in mice overexpressing DGAT in the liver. Cell Metab. 2007;6(1):69-78.

150.Chen HC, Stone SJ, Zhou P, Buhman KK, Farese RV Jr. Dissociation of obesity and impaired glucose disposal in mice overexpressing acyl coenzyme a: diacylglycerol acyltransferase 1 in white adipose tissue. Diabetes. 2002;51(11):3189-3195.

151.Koliwad SK, et al. DGAT1-dependent triacylglycerol storage by macrophages protects mice from diet-induced insulin resistance and inflammation. J Clin Invest. 2010;120(3):756-767.

152. Liu L, et al. DGAT1 expression increases heart triglyceride content but ameliorates lipotoxicity. J Biol Chem. 2009;284(52):36312-36323.

153.Paul A, Chang BH, Li L, Yechoor VK, Chan L. Deficiency of adipose differentiation-related protein impairs foam cell formation and protects against atherosclerosis. Circ Res. 2008;102(12):1492-1501.

154.Faber B, et al. Identification of genes potentially involved in rupture of human atherosclerotic plaque. Circ Res. 2001;89(6):547-554.

155.Accioly MT, et al. Lipid bodies are reservoirs of cyclooxygenase- 2 and sites of prostaglandin-E2 synthesis in colon cancer cells. Cancer Res. 2008; 68(6):1732-1740.

156. Miyanari $Y$, et al. The lipid droplet is an important organelle for hepatitis $\mathrm{C}$ virus production. Nat Cell Biol. 2007;9(9):1089-1097. 